SECTION 4. Computer science, computer engineering and automation.

Dinara Zhaksylykovna Shyrynkhanova

2 year magistr of the speciality "Information systems " Taraz State University named after M.Kh. Dulati, Kazakhstan dinara_27inf@mail.ru

Ulmeken Mukhitovna Smailova candidate of physical and mathematical Sciences, associate Professor, Branch of JSC National centre of improvement of qualification of «Orleu» Institute for professional development of Zhambyl region, Kazakhstan samilova_tarsu@mail.ru

Alexandr Nikolayevich Shevtsov candidate of technical Sciences, President of International Academy International Academy of Theoretical \& Applied Sciences, (USA, Sweden, Kazakhstan)

Shev AlexXXXX@mail.ru

\title{
THE ANALYSIS OF TESTS AND CORBA TECHNOLOGY
}

Abstract: A program is often made up of separate components - independent blocks of code that implement a certain logic, distributed across a network and can be reused many times. They are used as building blocks to create complex distributed applications. Consider one of the variants of a combination of programs for the analysis of tests with this technology related to the underlying object architecture for building distributed object software systems.

Key words: test, program, system, code analysis, description, unit schema.

\section{АНАЛИЗ ТЕСТОВ И ТЕХНОЛОГИЯ СОRВА}

Аннотация: Разработанные программы - зачастую состоят из отдельных компонентов - самостоятельные блоки программного кода, которые реализуют определенную логику, распределены по сети и могут быть использованы многократно. Они используются в качестве строчтельных блоков для создания сложных распределенных приложений. Рассмотрим один из вариантов сочетания программь для анализа тестов с данной технологией относящейся $\kappa$ базовым объектным архитектурам для создания распределенных объектных программных систем.

Ключевые слова: тест, программа, система, код, анализ, описание, блок схема.

Как известно [1-3] Common Object Request Broker Architecture (CORBA), которую развивает Консорциум OMG. Цель этой статьи - рассмотреть некоторые вопросы разработки программного обеспечения на основе объектных моделей, необходимого для предварительного анализа и исправления ошибок в сочетании с CORBA.

\section{Основные архитектурные принципы и задачи}

Основное назначение CORBA - поддержка разработки и развертывания сложных объектно-ориентированных прикладных систем. Любого отдельно взятого объектно-ориентированного языка недостаточно для написания распределенных вычислительных систем. Очень часто различные компоненты программной системы требуют реализации на разных языках и, возможно, разных аппаратных платформах. С помощью объектных моделей множество объектов приложения, в том числе и на 
различных платформах, взаимодействуют друг с другом и реализуют процессы, создавая видимость единого целого [2-4].

Функции CORBA - это функции промежуточного программного обеспечения объектной среды. Для того чтобы обеспечить взаимодействие объектов и их интеграцию в цельную систему, архитектура промежуточного уровня должна реализовать несколько базовых принципов.

- Независимость от физического размещения объекта. Компоненты программного обеспечения не обязаны находиться в одном исполняемом файле, выполняться в рамках одного процесса или размещаться на одной аппаратной системе.

- Независимость от платформы. Компоненты могут выполняться на различных аппаратных и операционных платформах, взаимодействуя друг с другом в рамках единой системы.

- Независимость от языка программирования. Различия в языках, которые используются при создании компонентов, не препятствуют их взаимодействию друг с другом.

CORBA - это клиент-серверные технологии, в которых функциональность объекта предоставляется клиенту посредством обращения к абстрактным интерфейсам. Интерфейс определяет набор методов, которые реализуют функции, присущие данному классу объектов. Интерфейс дает клиенту возможность только вызывать тот или иной метод, скрывая от него все детали его реализации.

Клиент получает доступ к объекту только путем вызова метода, определенного в интерфейсе объекта. Это означает, что реальные действия выполняются в адресном пространстве объекта, возможно, удаленном по отношению к процессу клиента. Сокрытие деталей реализации и позволяет в конечном итоге добиться слаженного взаимодействия компонентов в независимости от того, где и на какой платформе они реализованы и какой язык программирования для этого использовался.

В обеих технологиях взаимодействие между клиентским процессом и сервером объекта, то есть процессом, который порождает и обслуживает экземпляры объекта, использует механизм объектный вариант вызова удаленной процедуры (RPC, remote procedure call). Структура RPC - старейшей механизм из технологий промежуточного программного обеспечения. Механизм RPC реализует схему передачи сообщений, в соответствии с которой в распределенном клиент-серверном приложении процедураклиент передает специальное сообщение с параметрами вызова по сети в удаленную серверную процедуру, а результаты ее выполнения возвращаются в другом сообщении клиентскому процессу [4-7].

Для того чтобы реализовать эту схему, на стороне клиента и на стороне сервера поддерживаются специальные компоненты, носящие название клиентский и серверный суррогаты (client stub и server stub). Для того чтобы вызвать ту или иную функцию, клиент обращается к клиентскому суррогату, который упаковывает аргументы в сообщение-запрос и передает их на транспортный уровень соединения. Серверный суррогат распаковывает полученное сообщение и в соответствии с переданными аргументами вызывает нужную функцию, или нужный метод объекта, если речь идет об объектном варианте RPC. В CORBA клиентский суррогат не имеет специального названия, а серверный обозначают термином skeleton.

Параметры вызова могут формироваться в отличной от серверной языковой и операционной среде, поэтому на клиентский и серверный суррогаты возлагаются функции преобразования аргументов и результатов в универсальное, не зависящее от конкретной архитектуры представление. Тем самым достигается возможность взаимодействия клиента и сервера на различных платформах. 
Разработка клиентского суррогата и его алгоритмы работы [8-9].

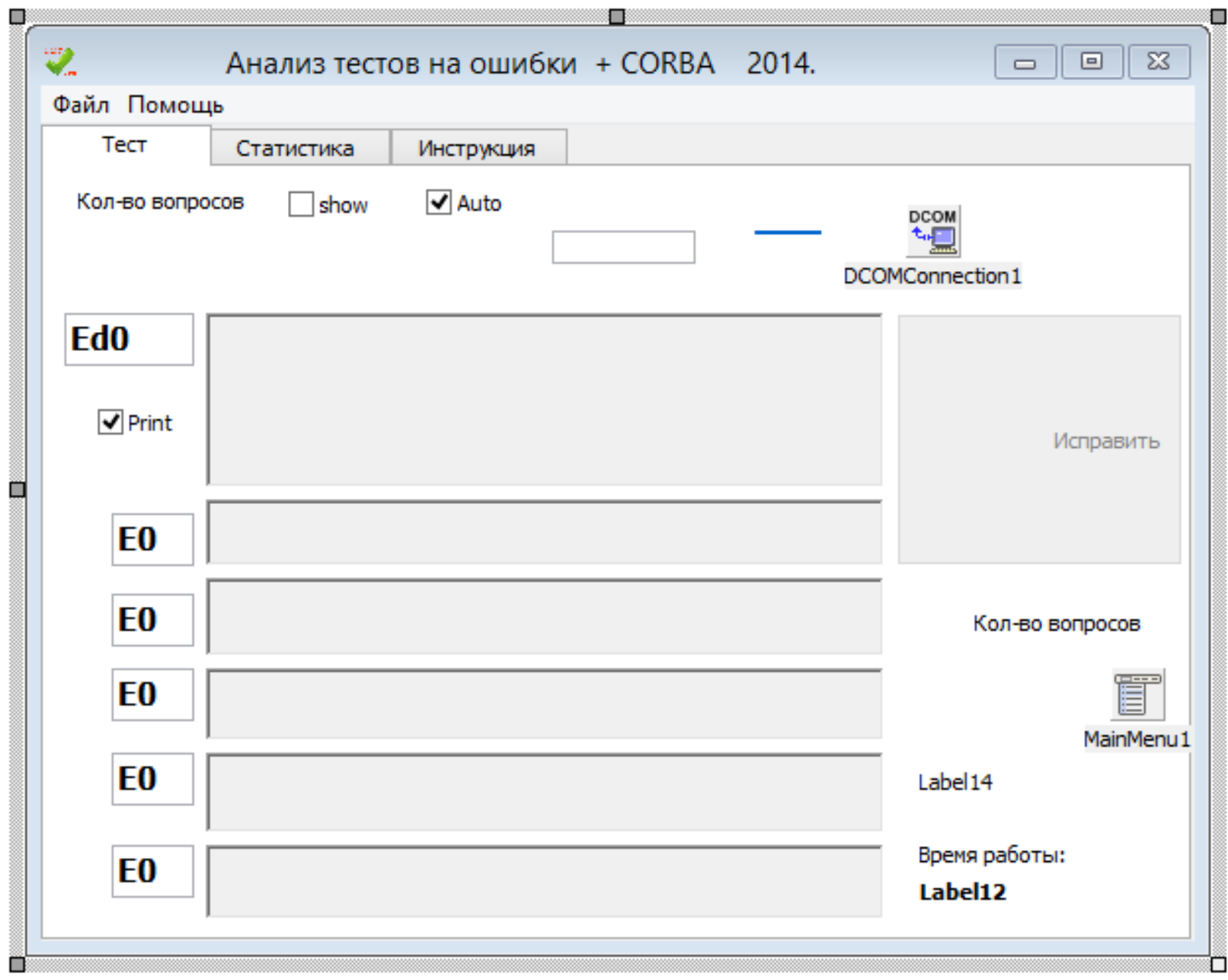

\section{Рисунок 1 - Клиентский суррогат.}

Назначение клиентского суррогата (рис.1) - предварительный анализ сдаваемого теста по какой -либо дисциплине бакалавриата или магистратуры для выявления, и автоматического исправления типичных ошибок.

Наиболее типичные ошибки встречающиеся в поступающих тестовых заданиях являются следующие:

- Наличие лишнего знака 9 (Enter), два и более подряд.

- Знак 9 (Enter) находится между вариантами ответов.

- Два правильных ответа.

- Ошибочная нумерация вопросов.

- Отсутствие точки после номера вопроса.

- Отсутствие скобки после буквы(индекса) ответа.

- Два одинаковых индекса ответов.

- Вопрос разбит на две строки знаком ๆ (Enter)

- Лишние пробелы в зоне индексов вопросов и ответов.

- Повторяющиеся вопросы в тесте.

- А также некоторые другие... 
Изучая и реализовывая различные варианты [3-5] алгоритмов анализа, мы пришли к выводу, что наилучшим вариантом будет:

- Абстрагироваться от теста, и сосредоточиться на структуре,

- Разработать для каждой ошибки свой отдельный алгоритм (каждая ошибка уникальна),

- Искать ошибки за один проход теста (минимизировать проходы),

- Не исправлять ошибки, а создать новый документ и набирать новый тест на основе исходного,

- Исправлять не орфографию, а структуру теста,

- Выделять характерные ошибки разных типов разными цветами,

- Сохранять результаты работы (отчет) посредством технологии corba на головной компьютер.

Для анализа структуры теста, как показали исследования, лучшим вариантом работы - будет использование технологии Ole и внедрение для контроля и временного хранения готовых OleContainer-ов (рис.1) в сочетании с соединением с сервером Word, где и будет осуществляться непосредственная обработки данных.

\section{Алгоритмы анализа структуры}

Процесс загрузки теста осуществляется технологией DragandDrop, и по еe завершении нажимаетмя копка «Исправить».

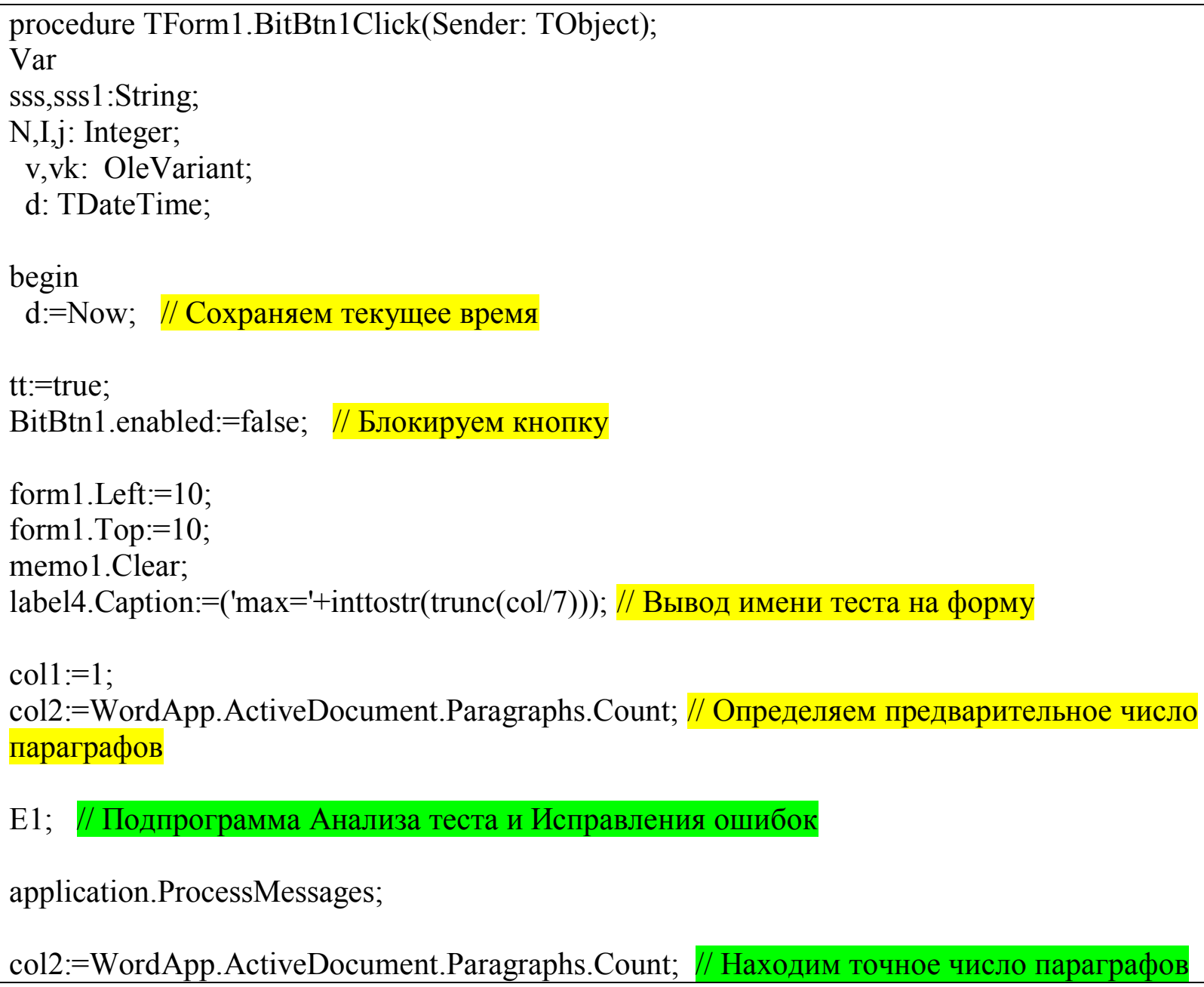




\section{// Выводим статистические результаты}

memol.lines.add('Общее количество ошибок = '+inttostr(memo1.lines.count));

label3.caption:='Общее количество ошибок $=$ '+inttostr(memo1.lines.count-1);

label10.caption: $=$ ' $\mathrm{N}=$ '+inttostr( $\operatorname{col} 2 \operatorname{div} 7)$;

memo1.lines.savetofile('Статистика ошибок теста - '+extractfilename(filename) +'.txt');

WordApp.ScreenUpdating := true;

WordApp1.ScreenUpdating $:=$ true;

Label12.Caption:=FormatDateTime('hh:mm:ss', Now()-d);

end;

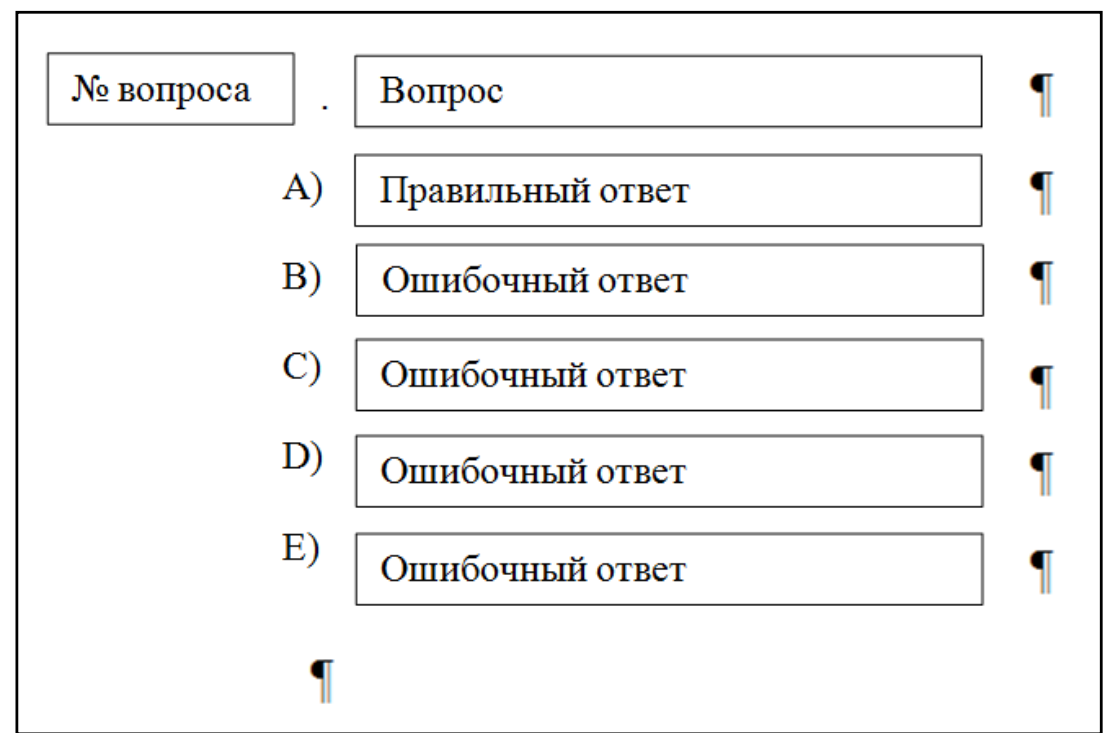

Рисунок 2 - Блок схема тестового задания.

Подпрограмма Анализа теста и Исправления ошибок

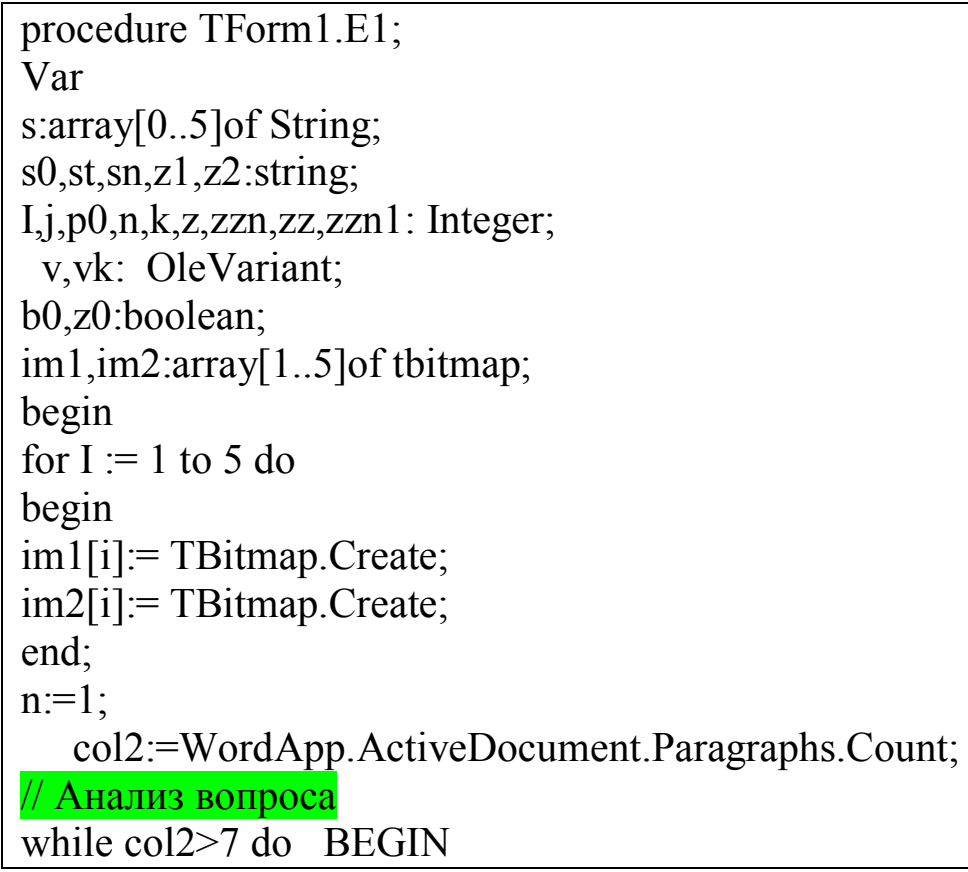




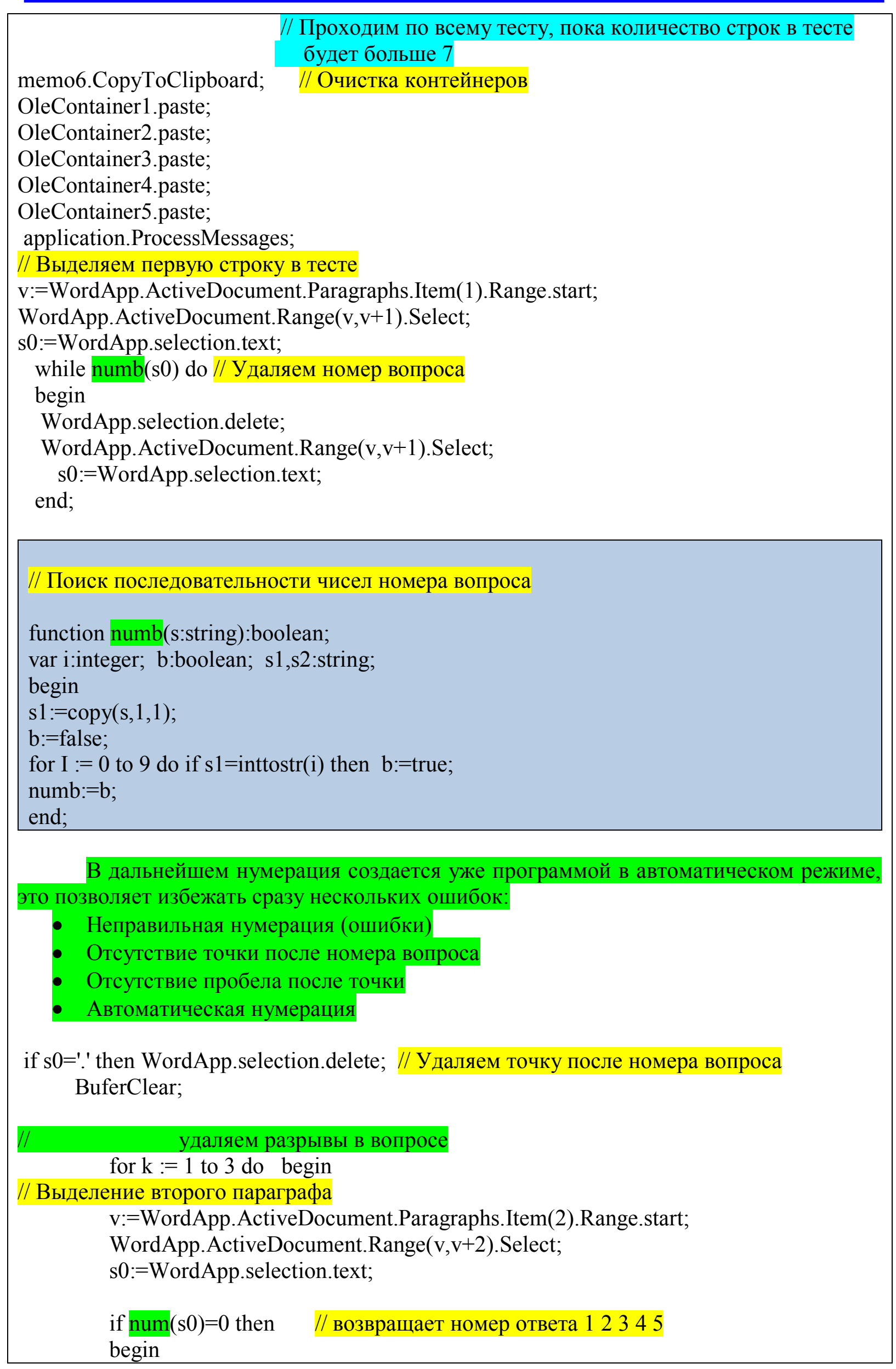


vk:=WordApp.ActiveDocument.Paragraphs.Item(1).Range.end;

WordApp.ActiveDocument.Range(vk-1,vk).Select;

WordApp.selection.delete;

end;

end;

// Определение номера вопроса

unction num(s:string):integer;

var i:integer; s1,s2:string;

begin

$\mathrm{s} 1:=\operatorname{copy}(\mathrm{s}, 1,1) ; \mathrm{s} 2:=\operatorname{copy}(\mathrm{s}, 2,1)$;

$\mathrm{i}:=0$;

if $\mathrm{s} 1={ }^{\prime}$ ' then $\mathrm{s}:=\mathrm{s} 2$ else $\mathrm{s}:=\mathrm{s} 1$;

if $\left(s={ }^{\prime} A^{\prime}\right)$ or $\left(s={ }^{\prime} A^{\prime}\right)$ then $i:=1$;

if $\left(\mathrm{s}^{\prime}{ }^{\prime} \mathrm{B}\right)$ or $\left(\mathrm{s}={ }^{\prime} \mathrm{B}\right)$ then $\mathrm{i}:=2$;

if $\left(s={ }^{\prime} C^{\prime}\right)$ or $\left(s={ }^{\prime} C^{\prime}\right)$ then $i:=3$;

if $\left(s^{\prime}{ }^{\prime} \mathrm{D}^{\prime}\right)$ or $\left(\mathrm{s}^{\prime}\right.$ 'Д') then $\mathrm{i}:=4$;

if $\left(s^{\prime}{ }^{\prime} E^{\prime}\right)$ or $\left(s^{\prime}{ }^{\prime} E^{\prime}\right)$ then $i:=5$;

num: $=\mathrm{i}$;

end;

WordApp.ActiveDocument.Paragraphs.Item(1).Range.select;

WordApp.selection.copy;

if checkbox2.checked then

OleContainer0.paste; // Вывод вопроса на экран

Ed0.Text:=inttostr(n)+'.'; // Вывод номера вопроса на экран

WordApp.selection.delete;

\section{/ Вывод номера вопроса в исправленный тест}

WordApp1.Selection.EndKey(wdStory,EmptyParam);

WordApp1.Selection.InsertAfter(inttostr(n)+'. ');

\section{/ Вывод вопроса в исправленный тест}

WordApp1.Selection.EndKey(wdStory,EmptyParam);

WordApp1.selection.paste;

inc(n); // Увеличение индекса вопросов на единицу вверх

application.ProcessMessages;

$\mathrm{z} 1:=" ; \quad \mathrm{zzn}:=0$;

$\mathrm{z} 2:="$;

\section{// Анализ теста и выделение ответов}

for $\mathrm{z}:=1$ to 5 do begin

BuferClear;

$\mathrm{v}:=$ WordApp.ActiveDocument.Paragraphs.Item(1).Range.start; 


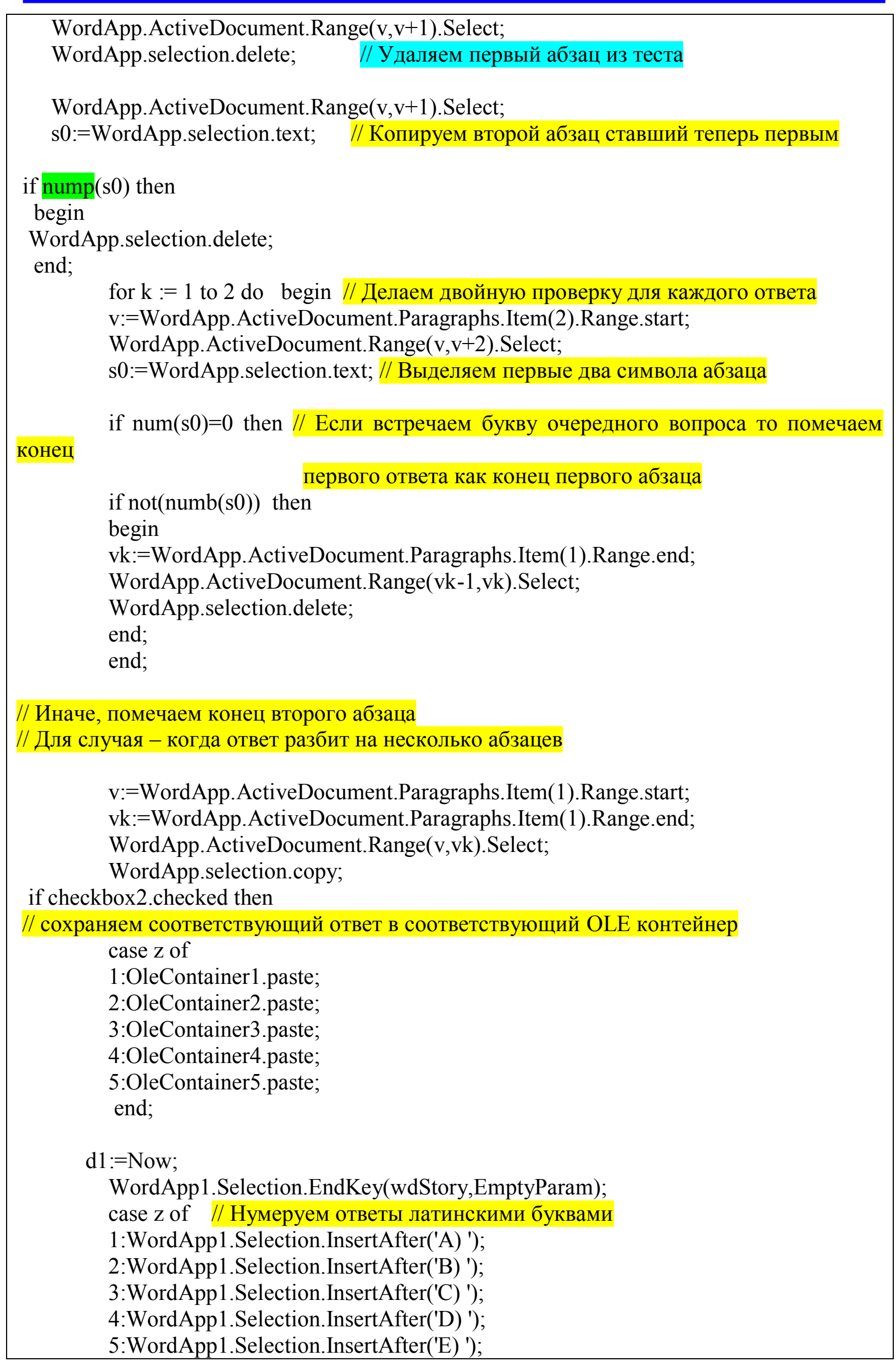


end;

memo5.lines.add(FormatDateTime('zzz',Now()-d1));

WordApp1.Selection.EndKey(wdStory,EmptyParam);

if numb(s0)and $(\mathrm{z}<5)$ then // Если одного из ответов нет - то сохраняем запись begin

WordApp1.Selection.InsertAfter(' //Нет ответа//'+\#13\#10);

WordApp.Selection.HomeKey(wdStory,EmptyParam);

WordApp.Selection.Insertbefore('A) '+\#13\#10);

$\mathrm{v}:=$ WordApp.ActiveDocument.Paragraphs.Item(2).Range.start;

WordApp.ActiveDocument.Range(v,v).Select;

WordApp.Selection.Insertbefore('A) ');

$\mathrm{v}:=$ WordApp.ActiveDocument.Paragraphs.Item(1).Range.start;

vk:=WordApp.ActiveDocument.Paragraphs.Item(1).Range.end;

WordApp.ActiveDocument.Range(v,vk).Select;

WordApp.selection.delete;

end

else

begin

$\mathrm{v}:=$ WordApp.ActiveDocument.Paragraphs.Item(1).Range.start;

vk:=WordApp.ActiveDocument.Paragraphs.Item(1).Range.end;

WordApp.ActiveDocument.Range(v,vk).Select;

WordApp1.selection.paste;

if $z=1$ then

begin

$\mathrm{z} 1:=$ WordApp.selection.text;

zzn:=WordApp.Selection.InlineShapes.count;

if $z z n>0$ then

for $\mathrm{zZ}:=1$ to $\mathrm{zzn}$ do

begin

WordApp.Selection.Range.InlineShapes.Item(zz).Select;//CopyAsPicture;

WordApp.Selection.Copy;

Im1[zz].Assign(Clipboard);

end;

end;

if $\mathrm{z}>1$ then

begin

$\mathrm{v}:=$ WordApp.ActiveDocument.Paragraphs.Item(1).Range.start;

vk:=WordApp.ActiveDocument.Paragraphs.Item(1).Range.end;

WordApp.ActiveDocument.Range(v,vk).Select;

z2:= WordApp.selection.text;

zzn1:=WordApp.Selection.InlineShapes.count;

if $z z n>0$ then

for $\mathrm{zz}:=1$ to $\mathrm{zzn} 1$ do

begin

WordApp.Selection.Range.InlineShapes.Item(zz).Select; 


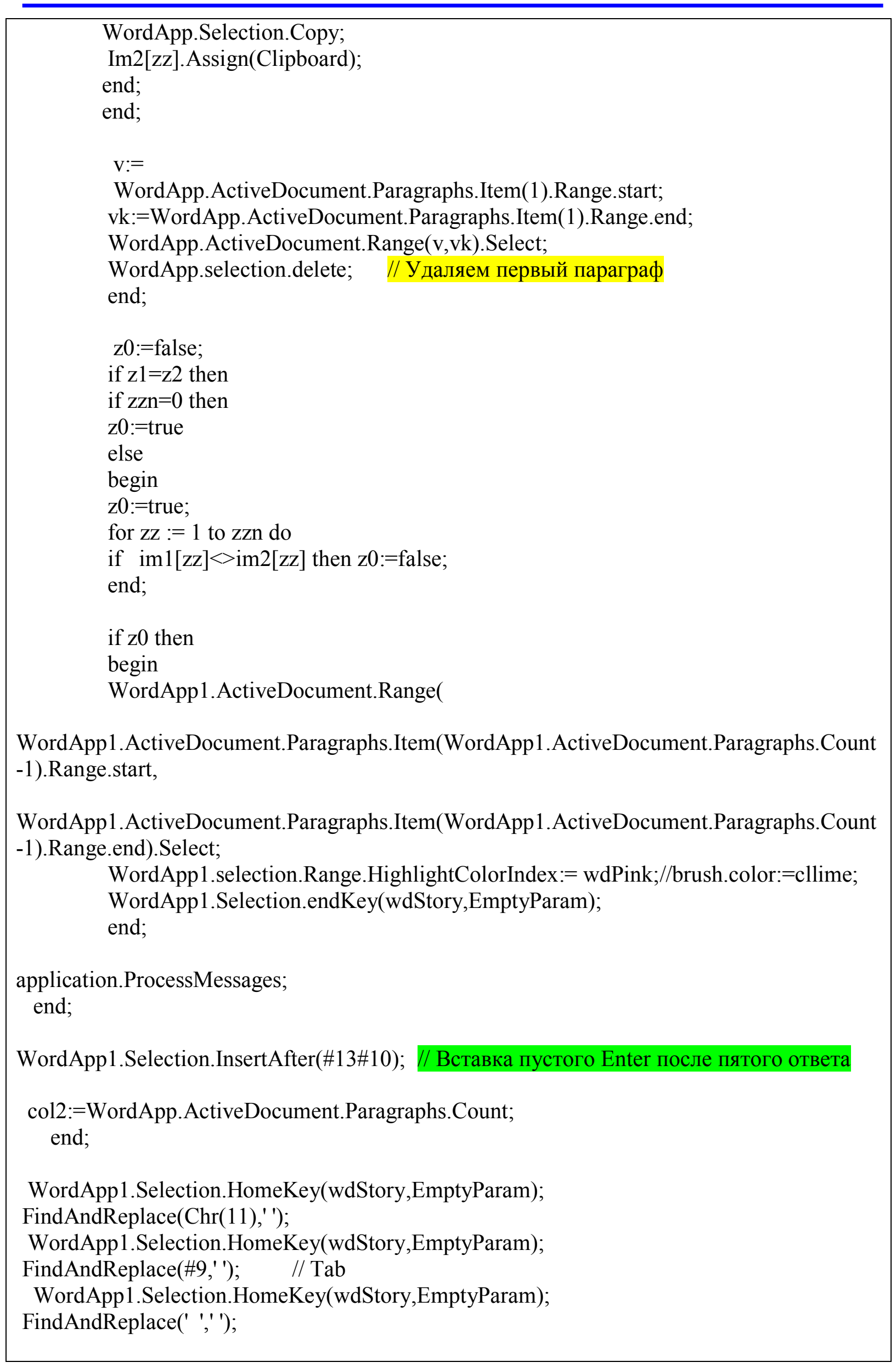




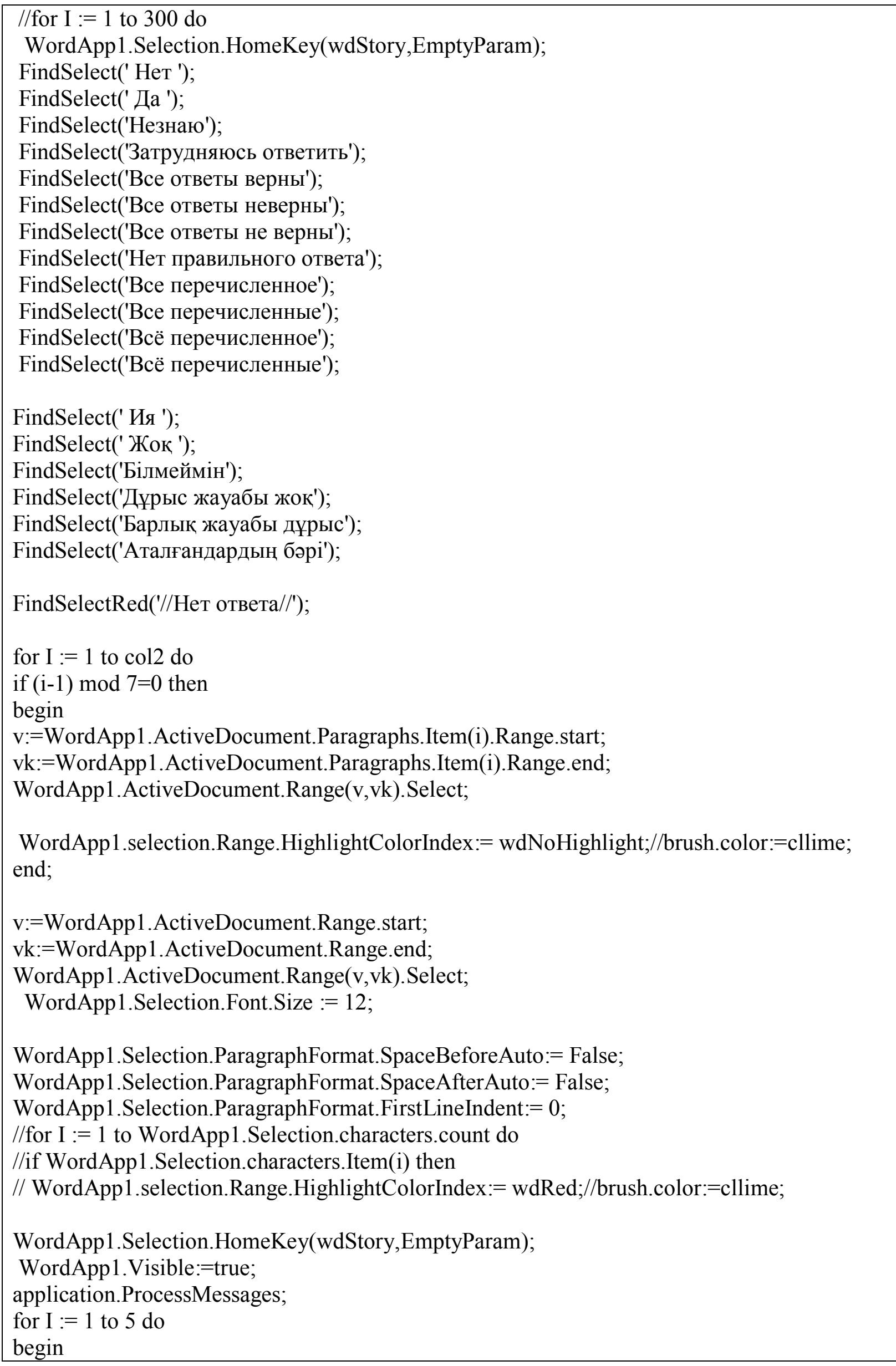


im1[i].free;

$\operatorname{im} 2[\mathrm{i}]$. free;

end;

end;

\section{References:}

1. Сравнение COM и CORBA [Электронный ресурс] URL: http://kunegin.com/ref3/corba5/12.htm (дата доступа 28.04.2014)

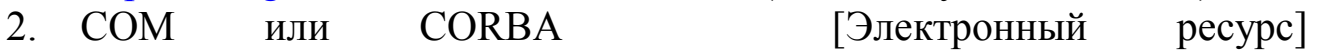

URL: http://delphiworld.narod.ru/base/com_or_corba.html (дата доступа 28.04.2014)

3. Механизм вызова удаленных процедур - RPC [Электронный ресурс] URL: http://delphiworld.narod.ru/base/rpc protocol.html (дата доступа 28.04.2014)

4. PowerBuilder 5.0 - открытый инструментарий для создания сложных распределенных клиент-серверных приложений [Электронный ресурс] URL: http://www.osp.ru/data/www2/dbms/1996/05/97.htm (дата доступа 28.04.2014)

5. Разработка распределённого Web-приложения [Электронный ресурc] URL: http://www.rsdn.ru/article/webdevelopment/49-57-distributedapps.xml (дата доступа 28.04.2014)

6. Понятие клиент-серверных систем [Электронный pecypc] URL: http://bourabai.kz/dbt/client1.htm (дата доступа 28.04.2014)

7. Компоненты сетевого приложения. Клиент-серверное взаимодействие и роли серверов. [Электронный ресурс] URL: http://www.4stud.info/networking/lecture5.html (дата доступа 28.04.2014)

8. Шевцов А.Н., Шырынханова Д.Ж. Разработка алгоритмов и приложения компонентной модели для анализа и исправления ошибок экзаменационного теста. Theoretical \& Applied Science. «Development of Applied Mathematics», ISPC, 30.05.2013, Taraz, Kazakhstan. - №5, 2013. -p.77-83. [Электронный pecypc] URL: http://elibrary.ru/item.asp?id=20357857 (дата доступа 28.04.2014)

9. Шевцов А.Н., Смайлова У.М., Шырынханова Д.Ж. Некоторые алгоритмы предварительной обработки теста. Theoretical \& Applied Science. «Results \& Perspectives», ISPC, 30.09.2013, Florence, Italy. - №9, 2013. -p.51-58. [Электронный pecypc] URL: http://elibrary.ru/item.asp?id=20362250 (дата доступа 28.04.2014)

10. Программа на Delphi «Тесты» [Электронный ресурс] URL: http://delphinew.ru/delphi-testi// (дата доступа 28.04.2014)

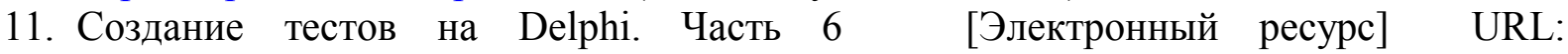
http://devdelphi.ru/?tag=\%d1\%82\%d0\%b5\%d1\%81\%d1\%82\%d1\%8b\%d0\%bd\%d0\%b0-delphi (дата доступа 28.04.2014)

12. Тестирование [Электронный http://www.helloworld.ru/texts/comp/lang/delphi/delphi4/test.htm 28.04.2014)

13. Уроки Delphi / Система проверки знаний. (оболочка теста на Delphi) [Электронный ресурс] URL: http://delphicomponent.ru/131-uroki-delphi-sistemaproverki-znaniy-obolochka-testa-na-delphi.html (дата доступа 28.04.2014) 